\title{
PLANEJAMENTO AGREGADO DA PRODUÇÃO EM NUTRIÇÃO DE BOVINOS DE CORTE: UMA ABORDAGEM ATRAVÉS DE PROGRAMAÇÃO LINEAR INTEIRA MISTA
}

\author{
Felipe Kesrouani Lemos ${ }^{1,3}$,Lilian Biazini de Moraes $^{2}$ \\ ${ }^{1}$ Docente, ${ }^{2}$ Discente do Curso de Engenharia de Produção da UNOESTE, Presidente Prudente, SP; ${ }^{3}$ Pós-Graduando em Engenharia \\ de Produção na Escola Politécnica da USP, São Paulo, SP. e-mail: felipeklemos@unoeste.br
}

\section{RESUMO}

Este artigo propõe um modelo de programação linear inteira mista de planejamento agregado de produção no contexto de fazendas de bovinocultura de corte em ciclo completo (cria, recria e engorda). Apesar das aplicações da pesquisa operacional no ramo ainda se restringirem a problemas de blending para formulação de rações, trata-se de uma abordagem útil e pertinente, sobretudo pela peculiaridade deste sistema de produção (em relação à indústria) de ter capacidade passível de alta variação ("sazonalidade da capacidade") ao longo do ano, devido às estações secas. Assim, o modelo apresentado tem o objetivo de auxiliar nas decisões de dimensionamento da quantidade de animais e, ao mesmo tempo, na forma de manejo dos pastos (decisões de veda e adubação) e manejo nutricional (decisões de suplementação e confinamento). O modelo foi testado com dados de uma agropecuária do oeste de São Paulo e Mato Grosso de Sul, através de entrevistas com gestores e trabalhadores rurais. Resultados interessantes para a gestão foram obtidos, possibilidade a utilização otimizada dos recursos naturais e um dimensionamento de orçamento antecipadamente. Os resultados preliminares motivaram a um maior refinamento dos parâmetros e abrem janelas para extensões da pesquisa incorporando novos elementos para enriquecer como ferramenta de apoio à decisão.

Palavras-chave: planejamento de produção, programação linear inteira mista, pecuária de corte, suplementação; adubação.

\section{MASTER PRODUCTION PLANNING ON BEEF CATTLE NUTRITION: A MIXED INTEGER LINEAR PROGRAMMING APPROACH}

\begin{abstract}
This paper proposes a mixed linear integer programming model of master production planning on beef cattle farms environment with complete cycle (breeding and finishing). In spite of operations research approach be restricted to blending problems to formulate diets, it's an useful and viable approach, mainly because of the special feature of this production system (comparing to factories) of having high variations of capacity ("capacity sazonality") during the year, due to dry stations. So, the model presented aims to support decisions of animal lots dimensions and, also, in the adoption of technologies for forages (intensification decisions) and nutrition (supplementation and feedlots use). The model was run with actual date of a company of western of São Paulo and Mato Grosso do Sul, through interviews with managers and workers. Interesting results were obtained, maing possible the optimized use of forage resources and budget dimension in advance. Preliminary results motivated to a new revision of parameters and open new fields to extend the research with new elements to empower the decision support tool.
\end{abstract}

Keywords: production planning, mixed integer linear programming, beef cattle, suplementation, forage intensification 


\section{INTRODUÇÃO}

O sistema agroindustrial (SAI) brasileiro é responsável por parcela importante da atividade econômica brasileira. Em 1988 empregava 23\% da população ativa, sendo responsável por mais de $30 \%$ do PIB (JANK, 1990). As agroindústrias alimentares representam um importante elo das cadeias produtivas que compõem este sistema (BATALHA, 1995). Em particular, na bovinocultura de corte, o Brasil se destaca como o maior país produtor e exportador. Seu sistema de produção está baseado no desenvolvimento de tecnologias de pastagens prioritariamente e da combinação deste mesmo sistema com os confinamentos de terminação de animais desde os anos 2000 (PIRES VAZ, 2010).

Pode-se definir um sistema de produção de gado de corte como um conjunto de tecnologias e práticas de manejo, tipos e raças de animais, objetivo da atividade e ambiente em que a atividade é realizada. Fazem parte da definição deste tipo de sistema os aspectos sociais, econômicos, culturais e mercadológicos (EUCLIDES FILHO, 2000).

A necessidade de aumento da competitividade no ramo é ressaltada por Siffert Filho e Faveret Filho (1998), que definem sistemas produtivos de alta performance como aqueles que buscam a maximização das economias de escala (operar no nível mínimo do custo médio), de escopo (combinar na mesma planta produtiva mais de um produto e/ou serviço) e de transação (redução dos custos de negociação).

O objetivo deste trabalho é modelar um sistema de produção de gado bovino de corte, possibilitando auxiliar nas decisões de planejamento de produção levando em conta suas restrições técnicas, alternativas tecnológicas e de produção. Utilizando a tipologia de metodologias quantitativas em modelagem e simulação de Morábito e Pureza (2010), esta pesquisa pode ser classificada como empírica prescritiva, já que apresenta como intuito a adesão entre a realidade deste sistema de produção e o modelo proposto; e ainda, busca apoiar o processo de decisões associadas ao planejamento de produção neste setor.

Como escopo desta pesquisa, pretendese estudar sistemas em que existem múltiplos locais de produção (fazendas), que possuem certo mix de espécies forrageiras onde os lotes de animais se alimentam continuamente. Estes pastos podem ser manejos de diversas formas (decisões de adubação e vedação para a seca), objetivando garantir as necessidades de matériaseca dos animais. Por outro lado, são possíveis diversos manejos nutricionais das categorias de engorda, oferecendo diferentes níveis de suplementação a pasto ou confinada para obter melhor performance de ganho de peso (aumentando o giro da propriedade). A Figura 1 ilustra o sistema e suas decisões destacadas em vermelho.

Assim, ao mesmo tempo em que este trabalho visa avaliar as decisões de produção quanto à ocupação dos recursos (pastagens); também se propõe a modelar a oportunidade de uso das tecnologias de intensificação, tanto no ramo da nutrição quanto da adubação de forrageiras. Uma característica interessante deste tipo de sistema é a alta sazonalidade da capacidade e não da carga, como é comum na indústria. A disponibilidade de pastagens é bastante reduzida no período da seca, o que leva a questões interessantes de como manter um rebanho com uma queda grande desta produção.

Este trabalho está organizado da seguinte maneira: após esta introdução que traz os objetivos, a apresentação do sistema em questão e conceitos iniciais da proposta da pesquisa, é apresentada uma breve revisão da literatura na seção 2; a seguir, é proposto um modelo matemático por programação linear inteira-mista 
para caracterizar o problema de planejamento da produção no tipo de sistema apresentado. Finalmente, na seção de resultados, são apresentados os métodos de coleta de dados, assim como os resultados obtidos rodando 0 modelo para uma agropecuária real com três propriedades. Nas sessões finais são dirigidas discussões e conclusões a respeito do método desenvolvido e dos resultados obtidos.

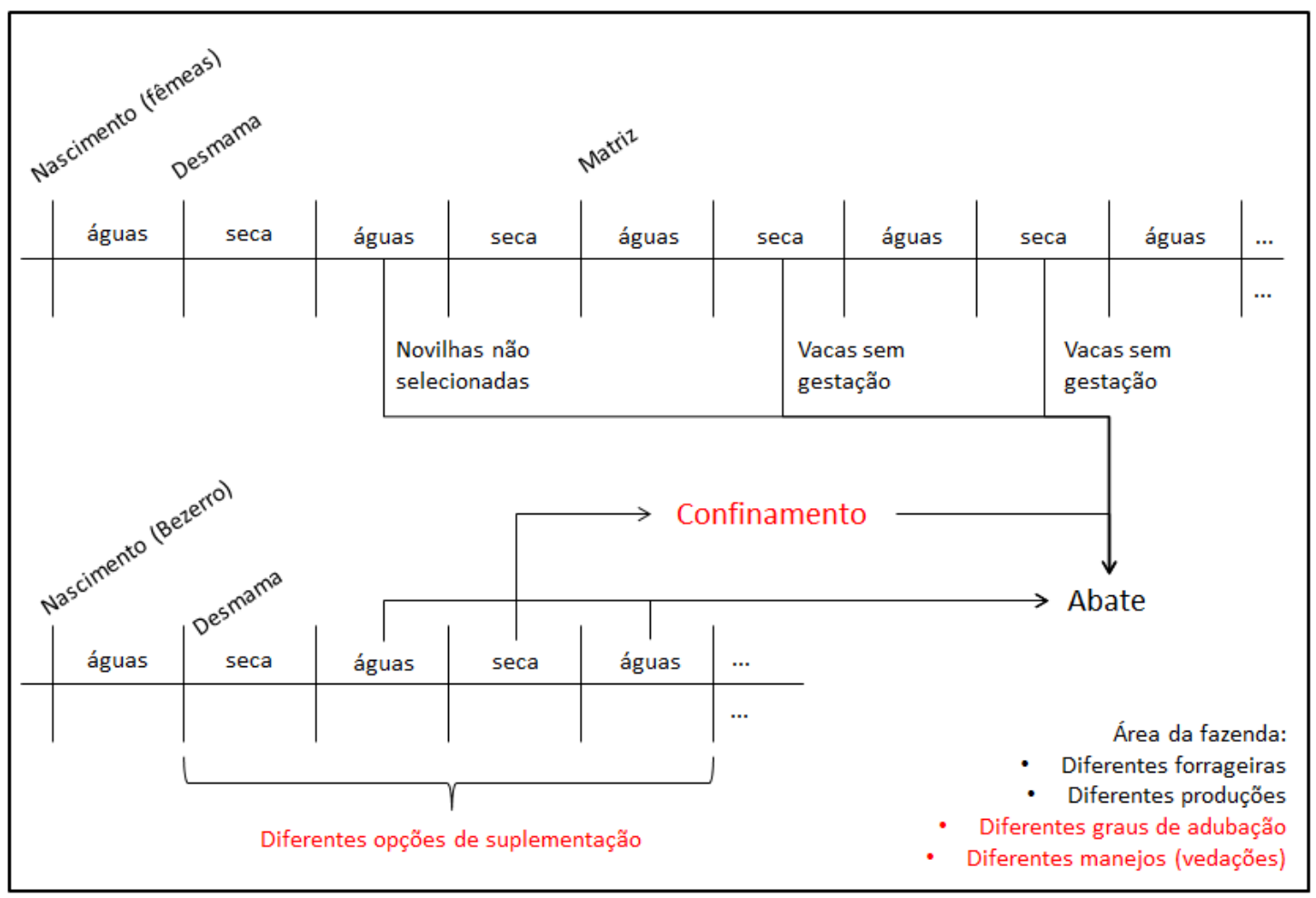

Figura 1. llustração de um sistema de produção de bovinocultura de corte.

\section{REVISÃO DA LITERATURA}

A utilização de técnicas de pesquisa operacional para a gestão da bovinocultura de corte ainda se mostra muito tímida na literatura. Sua faceta mais desenvolvida se dá na formulação de dietas para animais confinados, onde encontra-se um caso clássico do problema de blending. Lanna et al. (1996) promoveram a validação do sistema de estimativas de necessidades de nutrientes em gado de corte bovino da universidade de Cornell (NRC, 1996) em condições tropicais, o que culminou em um software adaptado às condições brasileiras (LANNA; TEDESCHI, 1998). Dumas et al. (2008) fazem uma revisão do uso de técnicas de modelagem na nutrição animal.
À parte de aplicações mais específicas, Ortuño e Vitoriano (2009) abordam o problema de planejamento na área rural através de uma modelagem em programação mista, porém com escopo amplo de escolha entre culturas no contexto espanhol. Outros modelos como estes são relativamente comuns na literatura, com escopo principalmente na agricultura. Siskos et al. (1993) propõem uma modelagem multi-objetivo no contexto da Tunísia; Darby-Dowman et al. (2000) endereçaram o problema da horticultura com componentes estocásticos; e Klein Haneveld e Stegeman (2005) modelaram o planejmento agrícola sob a ótica da sucessão de culturas.

Outras culturas agropecuárias também apresentam tímidas aplicações na área de 
pesquisa operacional. Dubeau et al. (2011) desenvolvem uma formulação linear para 0 problema de minimização do custo de dieta de suínos. Já Taube Netto (1996) propõe um modelo de planejamento integrado para a indústria do frango, focando a ligação das granjas com a indústria processadora.

No contexto de planejamento de produção propriamente dito, Derzko et al. (1980) propõem um modelo para otimizar a quantidade de animais que devem ser comprados, vendidos a abatidos a cada idade, de modo a maximizar os lucros do produtor. Já Moghaddam e DePuy (2011) abordam a produção de feno, sob a ótica de venda, produção e estocagem, considerando também questões probabilísticas relacionadas ao mercado e ao clima. Perpassando a ótica do produtor rural, Boyabatli et al. (2011) fazem uma modelagem integradora de decisões de curto e longo prazo com respeito à cadeia de suprimentos de carne bovina, porém sob a perspectiva da indústria processadora.

A respeito do uso de sistema de apoio à decisão no contexto rural, McCown et al. (2002) fazem uma revisão histórica de aplicações em diversas culturas, como algodão, trigo e também pecuária.

Reforçando a introdução desta seção, houve grande dificuldade de encontrar aplicações relacionadas ao planejamento da produção na área pecuária, em particular na bovinocultura de corte. Isso dá uma perspectiva exploratória a este estudo, na medida em que abre um campo de aprofundamento pouco explorado.

\section{METODOLOGIA}

\section{Formulação matemática}

Seja um sistema de produção bovinocultor constituído de $\mathrm{F}$ unidades produtivas, chamadas aqui de fazendas $(f=1, \ldots, F)$. Cada fazenda $f$ possui áreas de $\mathrm{J}$ diferentes forrageiras $(\mathrm{j}=1, \ldots, \mathrm{J})$, denotada por $A_{j f}$. Estas áreas podem ser submetidas a I diferentes tipos de manejos de pastagens $(i=1, \ldots, I)$, sendo que a cada tipo i de manejo de pastagens estão associadas capacidades de suporte por unidade de área em cada mês $\mathrm{t}$ do ano $(\mathrm{t}=1, \ldots, 12)$, parâmetro este denotado por $\lambda_{i j t}$. Estes tipos de manejos possíveis retratam as diferentes maneiras de adubação, bem como as decisões de vedar ou não uma determinada área em cada mês do ano. A cada tipo de manejo de pastagem $i$, na fazenda f, com o tipo de forrageira j, está associado também um custo variável por unidade de área, denotado por $C V_{i j f}^{p}$.

Cada tipo de manejo nutricional $k(k=1$, ..., K) em uma determinada fazenda $f$ leva a determinadas cargas em unidades animais (UA, sendo que $1 \mathrm{UA}=450 \mathrm{~kg}$ ) por matriz, em cada período $t$, parâmetro este denotado por $\theta_{k t}$. Por manejo logístico-nutricional, entende-se o conjunto de decisões que modulam a carga (em peso) de animais a que a fazenda estará submetida. Assim como no manejo de pastagem, cada manejo logístico-nutricional $\mathrm{k}$ tem um custo variável em relação ao número de matrizes, em uma dada fazenda f, denotado por $C V_{k f}^{b}$. Além do mais, cada manejo logístico-nutricional $\mathrm{k}$ em uma fazenda f implica uma receita por matriz $R_{k f}$.

Como variáveis de decisão deste modelo estão o número de matrizes submetidas a um determinado manejo logístico-nutricional $k$ na fazenda $\mathrm{f}, m_{k f}$; a área da forrageira $\mathrm{j}$ na fazenda f submetida ao manejo de pastagens i, $a_{i j f}$; e uma variável binária $\delta_{k}$, que vale 1 se o manejo logístico nutricional é escolhido e 0 caso contrário. Como parâmetro auxiliar, utiliza-se $U B m_{f}$ como um limitante superior do número de matrizes na fazenda $\mathrm{f}$. 


$$
\begin{aligned}
& \max _{i=1}=\sum_{k=1}^{K} \sum_{f=1}^{F} R_{k f} m_{k f}-\sum_{i=1}^{I} \sum_{j=1}^{J} \sum_{f=1}^{F} C V_{i j f}^{p} a_{i j f}-\sum_{k=1}^{K} \sum_{f=1}^{F} C V_{k f}^{b} m_{k f} \\
& \sum_{k=1}^{K} m_{k f} \theta_{k t} \leq \sum_{j=1}^{I} \sum_{i=1}^{I} a_{i j f} \lambda_{i j t}, \forall t, f \\
& m_{k f} \leq \delta_{k} U B m_{f}, \quad \forall k, f \\
& \sum_{k=1}^{K} \delta_{k}=1 \\
& \sum_{i=1}^{I} a_{i j f}=A_{j f}, \forall j f \\
& m_{k f}, a_{i j f} \in \mathbb{R}_{+}, \delta_{k} \in\{0,1\}
\end{aligned}
$$

O objetivo da formulação (1) é maximizar a margem, aqui retratada como a diferença entre a receita anual obtida através do manejo $\mathrm{k} e \mathrm{a}$ soma dos custos variáveis com bovinos, que é proporcional ao número de matrizes, e com pastagens, que é proporcional à área que recebe certo manejo. Interessante observar que está implícita nessa definição de margem que os demais custos (folha de pagamentos, manutenção de instalações, locomoção etc.) são fixos e estão mais relacionados com a área da fazenda (que não muda) do que ao tipo de manejo.

A restrição (2) é uma restrição típica de carga e capacidade, em que assegura-se que a carga total (em peso dos animais) em cada fazenda $f$, em cada mês $t$ não ultrapassa a sua capacidade (em capacidade de suporte das forrageiras). As restrições (3) e (4) garantem que só um tipo de manejo logístico-nutricional é adotado, forçando o número de matrizes nos demais ser zerado. Em (5), garante-se que a soma das áreas de uma forrageira j, em uma fazenda $f$ submetidas a diferentes manejos de pastagens i totaliza a área deste capim naquela propriedade. As restrições (6) definem a natureza das variáveis.

\section{Experimentos e resultados computacionais coleta de dados}

O modelo foi rodado utilizando os dados coletados de uma propriedade real, a Agropecuária A. Esta empresa possui três propriedades ( $f=1,2,3$ ), sendo que a primeira está situada no estado de SP e as duas outras no MS, conforme a Tabela 1.

Os coeficientes técnicos de carga dos manejos nutricionais $\left(\theta_{k t}\right)$ foram obtidos com base em simulações determinísticas. Foi feito um código em C para emular um sistema de produção com as premissas da Tabela 2. Assim, foi possível calcular quantas UAs são ocupadas para cada matriz da fazenda, considerando sua cria nos diferentes estágios da vida, com seus respectivos pesos. A Tabela 3 resume os coeficientes obtidos. 
Tabela 1. Áreas de cada espécie forrageira nas propriedades analisadas (em ha).

\begin{tabular}{|c|c|c|c|c|c|}
\hline & & & \multicolumn{3}{|c|}{ Fazenda } \\
\hline & & & 1 & 2 & 3 \\
\hline & & & SP & MS & MS \\
\hline \multirow{7}{*}{ Forrageira } & Brizantha & 1 & 500 & 1200 & 0 \\
\hline & Humidícola & 2 & 300 & 220 & 1250 \\
\hline & Brizantha / Decumbens & 3 & 0 & 80 & 0 \\
\hline & Mombaça & 4 & 150 & 650 & 0 \\
\hline & MG4 & 5 & 200 & 323 & 0 \\
\hline & Decumbens & 6 & 300 & 200 & 1550 \\
\hline & Humidícola / Decumbens & 7 & 0 & 0 & 2450 \\
\hline
\end{tabular}

Tabela 2. Premissas utilizadas na simulação determinística para obtenção dos coeficientes técnicos de carga em cada manejo nutricional $\left(\boldsymbol{\theta}_{\boldsymbol{k} t}\right)$.

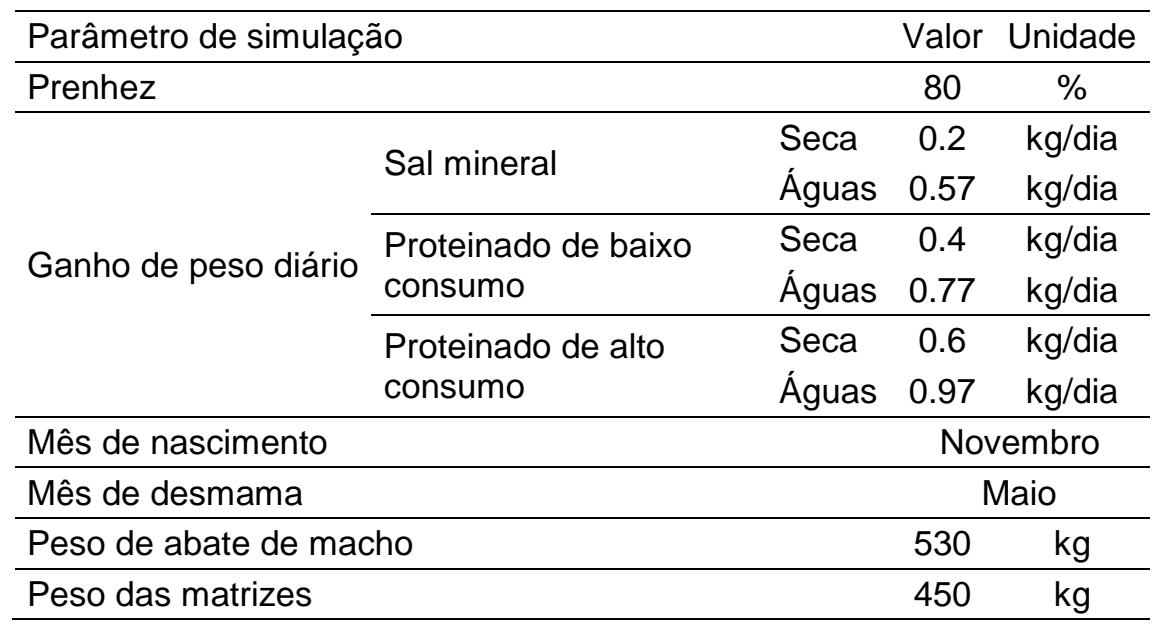

Tabela 3. Opções de manejo nutricional e suas respectivas cargas (em UA por matriz).

\begin{tabular}{|c|c|c|c|c|c|c|c|c|c|c|c|c|c|c|}
\hline \multirow{2}{*}{$\begin{array}{l}\text { Manejo } \\
\text { (k) }\end{array}$} & \multicolumn{2}{|c|}{ Caracterização do manejo } & \multicolumn{12}{|c|}{ Carga nos pastos (UA/matriz) - $\theta_{k t}$} \\
\hline & $\begin{array}{l}\text { Suplementação } \\
\text { a pasto }\end{array}$ & $\begin{array}{c}\text { Confinamento } \\
\text { na seca }\end{array}$ & 1 & 2 & 3 & 4 & 5 & 6 & 7 & 8 & 9 & 10 & 11 & 12 \\
\hline 1 & \multirow{2}{*}{ Sal mineral } & Sim & 2.4 & 2.4 & 2.5 & 2.6 & 1.9 & 1.9 & 2.0 & 2.0 & 2.0 & 2.0 & 2.2 & 2.3 \\
\hline 2 & & Não & 2.4 & 2.4 & 2.5 & 2.6 & 2.3 & 2.4 & 2.4 & 2.4 & 2.5 & 2.5 & 2.2 & 2.3 \\
\hline 3 & \multirow{2}{*}{$\begin{array}{l}\text { Proteinado de } \\
\text { baixo consumo }\end{array}$} & Sim & 2.0 & 2.1 & 2.2 & 2.2 & 1.6 & 1.6 & 1.7 & 1.7 & 1.7 & 1.7 & 1.9 & 2.0 \\
\hline 4 & & Não & 2.0 & 2.1 & 2.2 & 2.2 & 2.0 & 2.0 & 2.0 & 2.1 & 2.1 & 2.2 & 2.3 & 2.4 \\
\hline 5 & \multirow{2}{*}{$\begin{array}{l}\text { Proteinado de } \\
\text { alto consumo }\end{array}$} & Sim & 2.1 & 2.1 & 2.2 & 2.3 & 1.6 & 1.6 & 1.7 & 1.7 & 1.7 & 1.7 & 1.9 & 2.0 \\
\hline 6 & & Não & 2.0 & 2.1 & 2.2 & 2.2 & 2.0 & 2.0 & 2.0 & 2.1 & 2.1 & 2.2 & 2.3 & 2.4 \\
\hline
\end{tabular}


Já os coeficientes técnicos de capacidade dos manejos de pastagem $\left(\lambda_{i j t}\right)$ foram estimados com base em entrevistas. Como não existem dados de pesquisa consolidados sobre a disponibilidade de matéria seca para diversos tipos de manejo, optou-se por um questionário tríplice com um encarregado do campo, um gerente da empresa e um consultor de nutrição animal. Ao obter opções muito díspares, optou-se por realizar um painel para discussão até convergir em um consenso. A Tabela 4.4 resume as opções de manejo de pastagens consideradas e os respectivos coeficientes técnicos associados.

Finalmente, os parâmetros econômicos foram estimados com base em dados de mercado. Para chegar aos valores apresentados nas Tabelas 4.6 e 4.7 utilizou-se as premissas da Tabela 5 .

Tabela 4. Opções de manejo de pastagens e suas respectivas capacidades (em UA por matriz).

\begin{tabular}{|c|c|c|c|c|c|c|c|c|c|c|c|c|c|c|c|}
\hline \multirow{2}{*}{$\begin{array}{l}\text { Forrageira } \\
\text { (j) }\end{array}$} & \multirow{2}{*}{$\begin{array}{l}\text { Manejo } \\
\text { (i) }\end{array}$} & \multicolumn{2}{|c|}{$\begin{array}{c}\text { Caracterização do } \\
\text { manejo }\end{array}$} & \multicolumn{12}{|c|}{ Capacidade (UA/ha) - $\lambda_{i j t}$} \\
\hline & & Adubação & $\begin{array}{l}\text { Veda } \\
\text { para seca }\end{array}$ & 1 & 2 & 3 & 4 & 5 & 6 & 7 & 8 & 9 & 10 & 11 & 12 \\
\hline \multirow{4}{*}{1 e 5} & 1 & \multirow{2}{*}{ Sim } & Não & 3.0 & 3.0 & 3.0 & 3.0 & 1.0 & 1.0 & 1.0 & 1.0 & 1.0 & 3.0 & 3.0 & 3.0 \\
\hline & 2 & & Sim & 1.0 & 1.0 & 1.0 & 1.0 & 3.0 & 3.0 & 3.0 & 3.0 & 3.0 & 1.0 & 1.0 & 1.0 \\
\hline & 3 & \multirow{2}{*}{ Não } & Não & 1.5 & 1.5 & 1.5 & 1.5 & 0.5 & 0.5 & 0.5 & 0.5 & 0.5 & 1.5 & 1.5 & 1.5 \\
\hline & 4 & & Sim & 0.5 & 0.5 & 0.5 & 0.5 & 1.5 & 1.5 & 1.5 & 1.5 & 1.5 & 0.5 & 0.5 & 0.5 \\
\hline \multirow{4}{*}{2,6 e 7} & 1 & \multirow{2}{*}{ Sim } & Não & 1.5 & 1.5 & 1.5 & 1.5 & 0.8 & 0.8 & 0.8 & 0.8 & 0.8 & 1.5 & 1.5 & 1.5 \\
\hline & 2 & & Sim & 1.0 & 1.0 & 1.0 & 1.0 & 1.0 & 1.0 & 1.0 & 1.0 & 1.0 & 1.0 & 1.0 & 1.0 \\
\hline & 3 & & Não & 0.8 & 0.8 & 0.8 & 0.8 & 0.4 & 0.4 & 0.4 & 0.4 & 0.4 & 0.8 & 0.8 & 0.8 \\
\hline & 4 & INao & Sim & 0.5 & 0.5 & 0.5 & 0.5 & 0.5 & 0.5 & 0.5 & 0.5 & 0.5 & 0.5 & 0.5 & 0.5 \\
\hline \multirow{4}{*}{3} & 1 & & Não & 2.5 & 2.5 & 2.5 & 2.5 & 0.5 & 0.5 & 0.5 & 0.5 & 0.5 & 2.5 & 2.5 & 2.5 \\
\hline & 2 & & Sim & 1.0 & 1.0 & 1.0 & 1.0 & 1.0 & 1.0 & 1.0 & 1.0 & 1.0 & 1.0 & 1.0 & 1.0 \\
\hline & 3 & & Não & 1.3 & 1.3 & 1.3 & 1.3 & 0.3 & 0.3 & 0.3 & 0.3 & 0.3 & 1.3 & 1.3 & 1.3 \\
\hline & 4 & Nao & Sim & 0.5 & 0.5 & 0.5 & 0.5 & 0.5 & 0.5 & 0.5 & 0.5 & 0.5 & 0.5 & 0.5 & 0.5 \\
\hline \multirow{4}{*}{4} & 1 & \multirow{2}{*}{$\operatorname{Sim}$} & Não & 5.0 & 5.0 & 5.0 & 5.0 & 1.0 & 1.0 & 1.0 & 1.0 & 1.0 & 5.0 & 5.0 & 5.0 \\
\hline & 2 & & Sim & 1.0 & 1.0 & 1.0 & 1.0 & 1.0 & 1.0 & 1.0 & 1.0 & 1.0 & 1.0 & 1.0 & 1.0 \\
\hline & 3 & \multirow{2}{*}{ Não } & Não & 2.5 & 2.5 & 2.5 & 2.5 & 0.5 & 0.5 & 0.5 & 0.5 & 0.5 & 2.5 & 2.5 & 2.5 \\
\hline & 4 & & Sim & 0.5 & 0.5 & 0.5 & 0.5 & 0.5 & 0.5 & 0.5 & 0.5 & 0.5 & 0.5 & 0.5 & 0.5 \\
\hline
\end{tabular}

Tabela 5. Premissas utilizadas para o cálculo dos parâmetros econômicos.

\begin{tabular}{|c|c|c|c|c|}
\hline \multicolumn{3}{|l|}{ Premissas } & \multirow{2}{*}{$\begin{array}{l}\text { Valor } \\
1.13\end{array}$} & \multirow{2}{*}{$\begin{array}{l}\text { Unidade } \\
\mathrm{R} \$ / \mathrm{kg}\end{array}$} \\
\hline \multirow{8}{*}{ Nutrição } & \multirow[b]{2}{*}{ Sal mineral } & Preço & & \\
\hline & & Consumo diário & 0.22 & $\begin{array}{l}\text { g/dia/kg de peso } \\
\text { vivo }\end{array}$ \\
\hline & \multirow{2}{*}{$\begin{array}{l}\text { Proteinado de baixo } \\
\text { consumo }\end{array}$} & Preço & 0.74 & $\mathrm{R} \$ / \mathrm{kg}$ \\
\hline & & Consumo diário & 1.00 & $\begin{array}{l}\text { g/dia/kg de peso } \\
\text { vivo }\end{array}$ \\
\hline & \multirow{2}{*}{$\begin{array}{l}\text { Proteinado de alto } \\
\text { consumo }\end{array}$} & Preço & 0.74 & $\mathrm{R} \$ / \mathrm{kg}$ \\
\hline & & Consumo diário & 2.00 & $\begin{array}{l}\text { g/dia/kg de peso } \\
\text { vivo }\end{array}$ \\
\hline & \multicolumn{2}{|c|}{ Diária de confinamento } & 5.00 & $\mathrm{R} \$ / \mathrm{kg}$ \\
\hline & \multicolumn{2}{|c|}{ Ganho de peso diário no confinamento } & 1.50 & $\mathrm{~kg} / \mathrm{dia}$ \\
\hline Receitas & \multicolumn{2}{|c|}{ Aumento de preço no MS em relação a SP } & 2.50 & $\%$ \\
\hline
\end{tabular}




\begin{tabular}{|c|c|c|c|c|}
\hline & \multicolumn{2}{|c|}{ Valor da @ de macho em SP } & 99.00 & $\mathrm{R} \$$ \\
\hline & \multicolumn{2}{|c|}{ Diferencial de base para fêmeas } & 10.00 & $\mathrm{R} \$$ \\
\hline & \multicolumn{2}{|c|}{ Diferencial de base para MS } & 7.00 & $\mathrm{R} \$$ \\
\hline \multirow{8}{*}{ Pastagens } & \multirow{2}{*}{ Calcário } & Preço & 75 & $\mathrm{R} \$ / \mathrm{t}$ \\
\hline & & Quantidade & 1.5 & t/ha \\
\hline & \multirow{2}{*}{ Fosfato } & Preço & 1200 & $\mathrm{R} \$ / \mathrm{t}$ \\
\hline & & Quantidade & 0.15 & t/ha \\
\hline & \multirow{2}{*}{ Uréia } & Preço & 1080 & $\mathrm{R} \$ / \mathrm{t}$ \\
\hline & & Quantidade & 0.33 & t/ha \\
\hline & \multirow{2}{*}{$\mathrm{KCl}$} & Preço & 1000 & $\mathrm{R} \$ / \mathrm{t}$ \\
\hline & & Quantidade & 0.08 & t/ha \\
\hline
\end{tabular}

Tabela 6. Parâmetros econômicos relacionado com o manejo de pastagens.

\begin{tabular}{|c|c|c|}
\hline $\begin{array}{l}\text { Fazenda } \\
\text { (f) }\end{array}$ & $\begin{array}{l}\text { Manejo } \\
\text { (i) }\end{array}$ & CV \\
\hline \multirow{4}{*}{1} & 1 & $\mathrm{R} \$ 728,90$ \\
\hline & 2 & $\mathrm{R} \$ 728,90$ \\
\hline & 3 & $\mathrm{R} \$$ \\
\hline & 4 & $\mathrm{R} \$$ \\
\hline \multirow{4}{*}{2} & 1 & $\mathrm{R} \$ 747,12$ \\
\hline & 2 & $\mathrm{R} \$ 747,12$ \\
\hline & 3 & $\mathrm{R} \$$ \\
\hline & 4 & $\mathrm{R} \$$ \\
\hline \multirow{4}{*}{3} & 1 & $\mathrm{R} \$ 747,12$ \\
\hline & 2 & $\mathrm{R} \$ 747,12$ \\
\hline & 3 & $\mathrm{R} \$ \quad-$ \\
\hline & 4 & $\mathrm{R} \$$ \\
\hline
\end{tabular}

Tabela 7. Parâmetros econômicos relacionado com o manejo nutricional.

\begin{tabular}{|c|c|c|c|}
\hline $\begin{array}{l}\text { Fazenda } \\
\text { (f) }\end{array}$ & $\begin{array}{l}\text { Manejo } \\
\text { nutricional (k) }\end{array}$ & Receita $\left(\boldsymbol{R}_{\boldsymbol{k f}}\right)$ & $\begin{array}{l}\text { Custo Variável } \\
\left(\boldsymbol{C} \boldsymbol{V}_{\boldsymbol{k f f}}^{b}\right)\end{array}$ \\
\hline \multirow{6}{*}{1} & 1 & \multirow{6}{*}{$\mathrm{R} \$ 1,237.57$} & $\mathrm{R} \$ 211.70$ \\
\hline & 2 & & $\mathrm{R} \$ 98.45$ \\
\hline & 3 & & $\mathrm{R} \$ 269.03$ \\
\hline & 4 & & $\mathrm{R} \$ 130.38$ \\
\hline & 5 & & $\mathrm{R} \$ 233.27$ \\
\hline & 6 & & $\mathrm{R} \$ 195.52$ \\
\hline \multirow{6}{*}{2} & 1 & \multirow{6}{*}{$\mathrm{R} \$ 1,146.24$} & $\mathrm{R} \$ 216.99$ \\
\hline & 2 & & $\mathrm{R} \$ 100.91$ \\
\hline & 3 & & $\mathrm{R} \$ 453.02$ \\
\hline & 4 & & $\mathrm{R} \$ 310.90$ \\
\hline & 5 & & $\mathrm{R} \$ 613.29$ \\
\hline & 6 & & $\mathrm{R} \$ 574.60$ \\
\hline
\end{tabular}




\begin{tabular}{lll}
\hline 3 & $\frac{R}{2}$ & $\frac{R}{R} \$ 160.99$ \\
\hline 3 & $\frac{R}{3}$ & $\frac{R}{R} \$ 353.02$ \\
& $\frac{R}{5}$ & $\mathrm{R} \$ 610.90$ \\
\hline
\end{tabular}

\section{RESULTADOS}

O modelo proposto foi implementado em CPLEX 12.3 com interface excel e rodado em um computador com processador i7 $2^{\underline{a}}$ geração, com memória RAM $8 \mathrm{~Gb}$. O tempo de processamento foi praticamente instantâneo, de forma que não se trata de um dado limitante (indicativo de que podem ser rodadas instâncias maiores).

Como pode ser visto na Tabela 8 , o modelo indica que em todas as propriedades deve-se optar pelo manejo 5 (proteinado de alto consumo com confinamento para terminação na seca) como nutrição da engorda. Quanto às pastagens (Tabela 9), os resultados são interessantes, na medida em que dão um panorama do mix de forrageiras que devem ser manejadas com veda ou sem veda em cada propriedade, além das oportunidades de adubação.

Tabela 8. Resultados obtidos no manejo nutricional: quantidade de matrizes por tipo de manejo.

\begin{tabular}{llcccc}
\hline \multirow{2}{*}{ Manejo } & \multirow{2}{*}{ Suplementação } & \multirow{2}{*}{ Confinamento } & \multicolumn{3}{c}{ Fazenda } \\
\cline { 3 - 5 } & & 1 & 2 & 3 \\
\hline 1 & \multirow{2}{*}{ Sal mineral } & Sim & 0 & 0 & 0 \\
\cline { 3 - 5 } & Não & 0 & 0 & 0 \\
\hline 3 & $\begin{array}{l}\text { Proteinado de baixo } \\
\text { consumo }\end{array}$ & Sim & 0 & 0 & 0 \\
\cline { 3 - 5 } & Não & 0 & 0 & 0 \\
\hline 4 & $\begin{array}{l}\text { Proteinado de alto } \\
\text { consumo }\end{array}$ & Sim & 636 & 1402 & 2464 \\
\cline { 3 - 5 } & & Não & 0 & 0 & 0 \\
\hline 6 & & & &
\end{tabular}

Tabela 9. Resultados obtidos no manejo de pastagens: áreas submetidas a cada tipo de manejo por fazenda (ha).

\begin{tabular}{|c|c|c|c|c|c|c|c|c|c|}
\hline \multirow{2}{*}{ Faz } & \multirow{2}{*}{ Adubação } & \multirow{2}{*}{$\begin{array}{l}\text { Vedação nas } \\
\text { águas }\end{array}$} & \multicolumn{7}{|c|}{ Forrageiras } \\
\hline & & & 1 & 2 & 3 & 4 & 5 & 6 & 7 \\
\hline \multirow{4}{*}{1} & \multirow{2}{*}{ Sim } & Não & 0.0 & 0.0 & 0.0 & 0.0 & 0.0 & 0.0 & 0.0 \\
\hline & & Sim & 0.0 & 0.0 & 0.0 & 0.0 & 0.0 & 0.0 & 0.0 \\
\hline & \multirow{2}{*}{ Não } & Não & 273.4 & 300.0 & 0.0 & 150.0 & 0.0 & 300.0 & 0.0 \\
\hline & & Sim & 226.6 & 0.0 & 0.0 & 0.0 & 200.0 & 0.0 & 0.0 \\
\hline \multirow{4}{*}{2} & \multirow{2}{*}{ Sim } & Não & 0.0 & 0.0 & 0.0 & 0.0 & 0.0 & 0.0 & 0.0 \\
\hline & & Sim & 0.0 & 0.0 & 0.0 & 0.0 & 0.0 & 0.0 & 0.0 \\
\hline & \multirow{2}{*}{ Não } & Não & 391.2 & 220.0 & 80.0 & 650.0 & 0.0 & 200.0 & 0.0 \\
\hline & & Sim & 808.8 & 0.0 & 0.0 & 0.0 & 323.0 & 0.0 & 0.0 \\
\hline \multirow{4}{*}{3} & \multirow{2}{*}{ Sim } & Não & 0.0 & 0.0 & 0.0 & 0.0 & 0.0 & 0.0 & 1974.8 \\
\hline & & Sim & 0.0 & 0.0 & 0.0 & 0.0 & 0.0 & 1550.0 & 475.2 \\
\hline & \multirow{2}{*}{ Não } & Não & 0.0 & 0.0 & 0.0 & 0.0 & 0.0 & 0.0 & 0.0 \\
\hline & & Sim & 0.0 & 1250.0 & 0.0 & 0.0 & 0.0 & 0.0 & 0.0 \\
\hline
\end{tabular}


A margem final obtida foi de $\mathrm{R} \$$ 2.509.631,77. Trata-se de um dado de difícil comparação com a situação atual da empresa, já que é uma margem, na qual ainda devem ser descontados os demais custos fixos. Como não houve acesso aos demais dados da empresa, a comparação não foi completamente possível.

\section{DISCUSSÃO}

Em uma nova rodada em discussões com a gestão da empresa, verificou-se que, mais do que os resultados finais, o modelo pode ser considerado um grande instrumento de gestão, sobretudo nas decisões de planejamento de médio prazo: plano de suplementação, adubação e reserva de pastos para a seca. Em particular, foi ressaltada com grande ânimo a utilidade desta última, já que as duas primeiras em geral podem ser avaliadas com pouca imprecisão através de técnicas de engenharia econômica (a imprecisão decorre da necessidade de utilizar dados médios de lotação e carga ao longo do ano). Entretanto, a escolha das áreas a serem vedadas para seca em geral é arbitrária e conta somente com o conhecimento tácito dos trabalhadores rurais. Importante ressaltar a delicadeza desta decisão, já que a falta de reservas pode levar a uma degradação das pastagens, cujo custo de reforma é muito significativo. Um fato interessante observado é justamente a forma como o resultado faz para balancear a questão peculiar da sazonalidade da capacidade do sistema.

Além disto, o modelo permite dimensionar os lotes de matrizes em cada propriedade, o que em geral também é feito de maneira intuitiva através de uma estimativa da disponibilidade de capim ao longo do ano.

Desta forma, trata-se de um modelo que concilia tanto decisões referentes a adoção de tecnologias para intensificação, quanto decisões operacionais de quantos lotes manter na propriedade e como manejar a reserva de pastagens.

Os resultados obtidos são preliminares, uma vez que é possível melhorar as estimativas de capacidade das forrageiras sob diferentes manejos através de pesquisas científicas de produção de matéria-seca. Além disso, componentes probabilísticos podem ser acrescentados para medir os impactos de alterações climáticas. Outro ponto importante são premissas simplificadoras para a geração dos parâmetros, como os nascimentos concentrados em um só mês, que podem ser melhorados.

\section{CONCLUSÃO}

O objetivo deste trabalho é modelar um sistema de produção de bovinocultura de corte, contemplando alternativas tecnológicas e de manejo em termos de pastagens e nutrição. Foi proposta uma formulação linear mista, com capacidade de solução em tempos bastante razoáveis, contemplando a questão do balanceamento entre carga e capacidade das propriedades nos diferentes cenários de manejo, maximizando a margem entre a receita e os custos decorrentes destas decisões.

Este estudo preliminar possibilitou o desenvolvimento do método, que mostra-se útil no apoio a decisões táticas e operacionais. Um refino deste estudo é necessário para melhorar os parâmetros de entrada do modelo.

Como sugestões de extensões desta pesquisa, é possível desenvolver análises de sensibilidade para indicar a que preço de insumos é compensatório utilizar cada uma das tecnologias enumeradas; permitir a recria e engordados machos em propriedades diferentes das de cria; e também a inserção de componentes probabilístico para simular incertezas climáticas e de mercado. 


\section{REFERÊNCIAS}

BATALHA, M. O. As cadeias de produção agroindustriais: uma perspectiva para o estudo das inovações tecnológicas. Revista de Administração, Vol. 30, n. 4, p. 43-50, 1995.

BOYABATLI, O.; KLEINDORFER PAUL, R. \& KOONTZ STEPHEN, K. Integrating Long-Term and Short-Term Contracting in Beef Supply Chains. Management Science, Vol. 57, n. 10, p. 1771-1787, http://dx.doi.org/10.1287/mnsc. 1110.1362

DARBY-DOWMAN, K.; BARKER, S.; AUDSLEY, E. \& PARSONS, D. A two stage stochastic programming with recourse model for determining robust planting plans in horticulture. Journal of the Operational Research Society, Vol. 51, p. 83-89, 2000.

DERZKO, N.; SETHI, S. P. \& THOMPSON, G. L. Distributed parameter systems approach to the optimal cattle ranching problem. Optimal Control Applications and Methods, Vol. 1, n. 1, p. 3-10, 1980.

http://dx.doi.org/10.1002/oca.4660010103

DUBEAU, F.; JULIEN, P.-O. \& POMAR, C. Formulating diets for growing pigs: economic and environmental considerations. Annals of Operations Research, Vol. 190, n. 1, p. 239-269, 2011.

http://dx.doi.org/10.1007/s10479-009-0633-1

DUMAS, A.; DIJKSTRA, J. \& FRANCE J. Mathematical modelling in animal nutrition: a centenary review. The Journal of Agricultural Science, Vol. 146, n. 2, p. 123-142, 2008. http://dx.doi.org/10.1017/S0021859608007703

EUCLIDES FILHO, K. Produção de bovinos de corte e o trinômio genótipo - ambiente mercado. Campo Grande: Embrapa Gado de Corte, 2000.61 p.

JANK, M. A inserção do Brasil e do setor cooperativo no mercado mundial de produtos agroindustrializados. São Paulo: ESALQ, 1990.

KLEIN HANEVELD, W. K. \& STEGEMAN, A. W. Crop succession requirements in agricultural production planning. European Journal of Operational Research, Vol. 166, p. 406-429, 2005.

http://dx.doi.org/10.1016/i.ejor.2004.03.009

LANNA, D. P. D. \& TEDESCHI, L. O. RLM 1.0 Sistema de cálculo de rações para lucro máximo e estimativa das exigências de bovinos de corte. Piracicaba: ESALQ, 1998.

LANNA, D. P. D.; FOX, D. G. \& BOIN, C. Validation of the Cornell Net Carbohydrate and
Protein System estimates of nutrient requirements of growing and lactating Zebu germplasm in tropical conditions. Journal of Animal Science, Vol. 74, p.287, 1996. McCown, R. L.; Hochman, Z. \& Carberry, P. S. Probing the enigma of the decision support system for farmers: Learning from experience and from theory. Agricultural Systems, Vol. 74, n. 1, p. 1-10.

MOGHADDAM, K. S. \& DEPUY, G. W. Farm management optimization using chance constrained programming method. Computers and Electronics in Agriculture, Vol. 77, n. 2, p. 229-237, 2011. http://dx.doi.org/10.1016/j.compag.2011.05.006

MORÁBITO, R. \& PUREZA, V. Modelagem e simulação, em: Metodologia de Pesquisa em Engenharia de Produção e Gestão de Operações, Paulo A. C. Miguel (ed), Editora Campus/Elsevier, Rio de Janeiro, 165-194, 2010.

Nacional Research Council. Nutrient requirements of beef cattle. $7^{\mathrm{a}}$ ed. Washington: Nacional Academy Press, 1996.

Ortu-o, M. T. \& Vitoriano, B. A goal programming approach for farm planning with resources dimensionality. Annals of Operations Research, Vol. 190, n. 1, p. 181-199, 2009. http://dx.doi.org/10.1007/s10479-009-0524-5

VAZ PIRES, A. Bovinocultura de corte. $1^{\underline{a}}$ ed. Piracicaba: FEALQ, 2010.

SIFFERT FILHO, N. \& FAVERET FILHO, P. O Sistema Agroindustrial de Carnes: Competitividade e Estruturas de Governança. Seminário sobre Competitividade na Indústria de Alimentos. Campinas: 1998.

SISKOS, Y., DESPOTIS, D. K., GHEDIRI, M. Multiobjective modelling for regional agricultural planning: case study in Tunisia. European Journal of Operational Research, Vol. 77, p. 375-391, 1993.

http://dx.doi.org/10.1016/0377-2217(94)90404-9

TAUBE NETTO, M. Integrated planning for poultry production at Sadia. Interfaces, Vol. 26, n. 1, p. 38-53, 1996.

http://dx.doi.org/10.1287/inte.26.1.38 